\title{
Characteristics of radiographic images acquired with CdTe, CCD and CMOS detectors in skull radiography
}

\author{
Polyane Mazucatto Queiroz $\mathbb{D}^{1, *}$, Gustavo Machado Santaella $\mathbb{D}^{1}$, Sergio Lucio Pereira de Castro Lopes $\mathbb{D}^{2}$, \\ Francisco Haiter-Neto(D) ${ }^{1}$, Deborah Queiroz Freitas $\mathbb{D}^{1}$ \\ ${ }^{1}$ Department of Oral Diagnosis, Division of Oral Radiology, Piracicaba Dental School, University of Campinas, Piracicaba, São Paulo, Brazil \\ ${ }^{2}$ Department of Diagnosis and Surgery, São José dos Campos Dental School, São Paulo State University, São José dos Campos, São Paulo, Brazil
}

\section{ABSTRACT}

Purpose: The purpose of this study was to evaluate the image quality, diagnostic efficacy, and radiation dose associated with the use of a cadmium telluride (CdTe) detector, compared to charge-coupled device (CCD) and complementary metal oxide semiconductor(CMOS) detectors.

Materials and Methods: Lateral cephalographs of a phantom (type 1) composed of synthetic polymer filled with water and another phantom (type 2) composed of human skull macerated with polymer coating were obtained with $\mathrm{CdTe}, \mathrm{CCD}$, and CMOS detectors. Dosimeters placed on the type 2 phantom were used to measure radiation. Noise levels from each image were also measured. McNamara cephalometric analysis was conducted, the dentoskeletal configurations were assessed, and a subjective evaluation of image quality was conducted. Parametric data were compared via 1-way analysis of variance with the Tukey post-hoc test, with a significance level of 5\%. Subjective image quality and dentoskeletal configuration were described qualitatively.

Results: A statistically significant difference was found among the images obtained with the 3 detectors $(P<0.05)$, with the lowest noise level observed among the images obtained with the CdTe detector and a higher subjective preference demonstrated for those images. For the cephalometric analyses, no significant difference $(P>0.05)$ was observed, and perfect agreement was seen with regard to the classifications obtained from the images acquired using the 3 detectors. The radiation dose associated with the CMOS detector was higher than the doses associated with the $\mathrm{CCD}(P<0.05)$ and CdTe detectors $(P<0.05)$.

Conclusion: Considering the evaluated parameters, the CdTe detector is recommended for use in clinical practice.

(Imaging Sci Dent 2020; 50: 339-46)

KEY WORDS: Noise; Diagnosis; Cephalometry

\section{Introduction}

As technology has advanced, devices with detectors have become commonly used for the acquisition of digital radiographic images. Compared to conventional films, digital detectors have the advantages of reduced working time, the possibility of image manipulation and duplication, a lower

This work was supported by the Coordination of Improvement of Higher Education Personnel, Brazil, under Finance Code 001.

Received March 30, 2020; Revised July 31, 2020; Accepted August 18, 2020

*Correspondence to : Prof. Polyane Mazucatto Queiroz

Department of Oral Diagnosis, Division of Oral Radiology, Piracicaba Dental School, 901 Limeira Avenue, Piracicaba, São Paulo 13414-903, Brazil

Tel) 55-19-2106-5327, E-mail) polyanequeiroz@ hotmail.com radiation dose, and easy storage. ${ }^{1,2}$ In dentistry, different types of digital detectors are often used for the acquisition of digital radiographic images, including the phosphor storage plate, charge-coupled device (CCD), and complementary metal oxide semiconductor (CMOS) detectors. ${ }^{3}$ Of these, the CCD and CMOS detectors are the most commonly used for the acquisition of extraoral images. ${ }^{4}$

The CCD and CMOS detectors consist of a silicon layer which, when exposed to X-ray radiation, undergoes a scintillation process. In this process, light emission causes the breakdown of covalent bonds in silicon, resulting in the release of electrons, which create an electric charge. This 
charge generation is proportional to the amount of radiation that arrives at the detector and, after an analog-digital conversion, yields a digital image. ${ }^{1,3}$

The detection of X-ray photons in CCD and CMOS systems is indirect due to the scintillation process. The indirect detection of the X-ray photons and subsequent steps involved with reading the electric charge can result in the loss of information and suboptimal use of the X-ray radiation. Therefore, researchers have sought to develop direct detectors. In direct detection, the X-ray photons are absorbed by a photoconductive layer and converted to electron-hole pairs. These pairs are collected in the form of electric charge and stored in capacitors, after which the digital radiographic image is formed. ${ }^{5}$

Direct detectors of X-ray photons require fewer complex structures than indirect detectors, resulting in lower manufacturing costs. ${ }^{5}$ Selenium detectors have been used for direct detection; however, this material exhibits low absorption of the X-ray photons. ${ }^{5}$ Cadmium telluride (CdTe), in turn, is characterized by components with a high atomic number, high density, and a high absorption capacity for $\mathrm{X}$-ray photons. As such, it is an alternative for the development of direct-sensing detectors that can theoretically provide higher-quality images using a lower radiation dose.,

Commercially, devices that include CdTe detectors already exist; however, no studies were found on the use of this technology in dentistry compared (with regard to different aspects) with CCD and CMOS detectors, which are already established in clinical practice and diagnosis.
Therefore, the present study was conducted with the objective of studying the image quality, diagnostic efficacy, and radiation dose associated with the CdTe detector in comparison with the CCD and CMOS detectors.

\section{Materials and Methods}

\section{Devices}

Lateral cephalographs were acquired using 3 devices, each with a different detector. The devices were 1) the ProMax 2S 2D (Planmeca, Helsinki, Finland) (CCD), 2) the Picasso Trio 3D (Vatech \& E-WOO Technology, Yongin, Republic of Korea) (CMOS), and 3) the Eagle 3D (Dabi Atlante, Ribeirão Preto, Brazil) (CdTe).

The acquisition parameters used for all images were standardized according to the exposure protocol for medium-size adults, which was calibrated by the manufacturer of each device. For CCD, this was $10 \mathrm{~mA}, 6.7 \mathrm{~s}$, and $66 \mathrm{kV}$; for CMOS, it was $9 \mathrm{~mA}, 12 \mathrm{~s}$, and $78 \mathrm{kV}$, and for CdTe, it was $8 \mathrm{~mA}, 10 \mathrm{~s}$, and $75 \mathrm{kV}$.

\section{Phantoms}

Two types of phantoms were used (Fig. 1) in this study. Type 1 was a synthetic polymer phantom shaped like an adult head (Equipandoloja Comercial, São Paulo, Brazil), filled with 2.5 liters of water and sealed with a pre-made rubber pin. This phantom was made from a store mannequin and was filled with distilled water to yield a more homogeneous internal density. The same amount of water
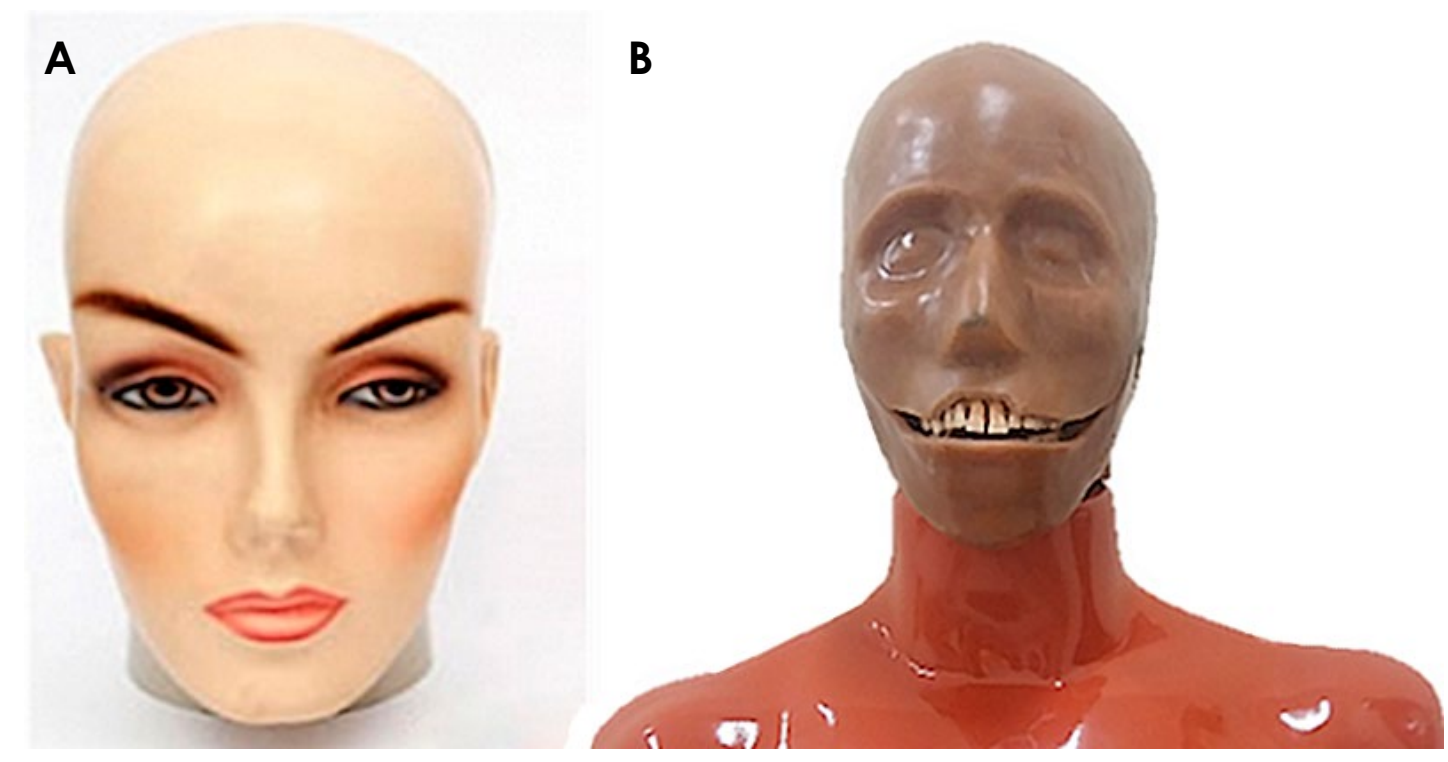

Fig. 1. A. The type 1 phantom is a synthetic polymer shaped like an adult head and filled with 2.5 liters of water. B. The type 2 phantom is made of a dry human skull coated with a synthetic polymer. 

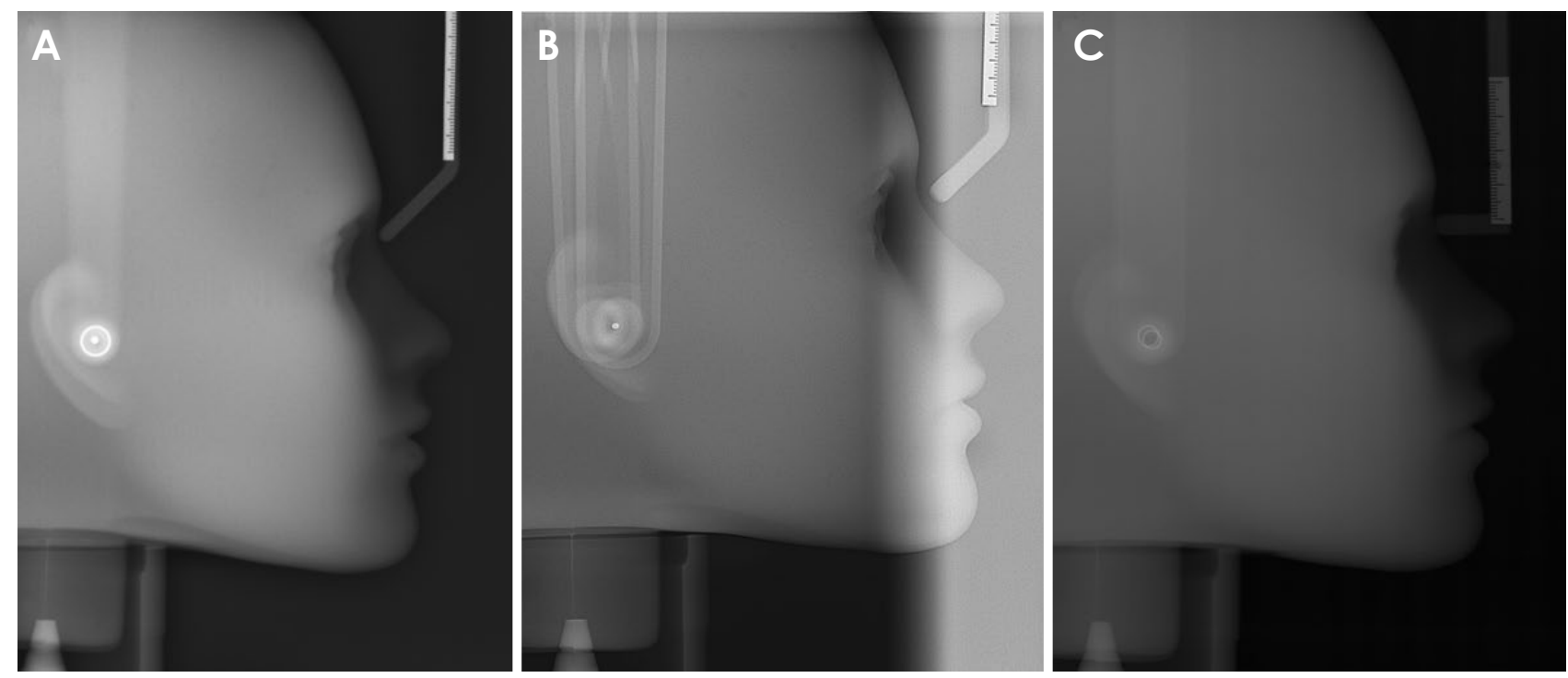

Fig. 2. Lateral cephalographs of the type 1 phantom for objective analysis acquired with charge-coupled device $(\mathrm{CCD}, \mathrm{A})$, complementary metal oxide semiconductor (CMOS, B), and cadmium telluride (CdTe, C) detector.

was used for all acquisitions.

The type 2 phantom was made of a dry human skull coated with a synthetic polymer (Nacional Osso, Jau, Brazil). This model of an advanced dental $\mathrm{X}$-ray trainer is used by dental students to practice intraoral imaging, and it was used in this study for cephalometric analysis and dosimeter positioning.

\section{Objective analysis of image quality}

The type 1 phantom was positioned in an acrylic resin base to standardize its positioning. Five images were acquired for each detector.

The images obtained (Fig. 2) were exported in TIFF format and evaluated in ImageJ software version 1.51i (U.S. National Institutes of Health, Bethesda, MD, USA). All images were individually calibrated using the provided ruler as the reference. Subsequently, a straight line (labeled R) was drawn adjacent to the anterior edge of the acrylic resin support and perpendicular to the horizontal plane of the radiograph. Perpendicular to line $\mathrm{R}$ and parallel to the horizontal plane, 5 lines were drawn (L1, L2, L3, L4, and L5) with reference to the cephalometric landmarks. L1 passed through the glabella point (in the soft tissue), L2 passed through the orbitale point (in the soft tissue), L3 passed through the pronasale point, L4 passed through the stomion point, and L5 passed through the B' point. Regions of interest (ROIs) measuring $10 \mathrm{~mm} \times 10 \mathrm{~mm}$ were selected in the areas in which line $\mathrm{R}$ touched the other lines. Using a magnification of $\times 5$, the ROIs were positioned adjacent to these reference lines, and the mean noise (standard deviation) values of each ROI were obtained, for a total of 5 ROIs per image (Fig. 3). This procedure was repeated after 15 days in $50 \%$ of the sample for the reproducibility evaluation, for which the intraclass correlation coefficient (ICC) was used.

The noise values were used to assess the homogeneity of the images. After the evaluation of the images acquired using the 3 detectors, the mean noise values of each detector were compared via 1-way analysis of variance (ANOVA) with the Tukey post-hoc test, using a significance level of 5\%. All statistical analyses were performed using BioEstat (Fundação Mamiraua, Belém, Brazil) and MedCalc 15.8 (MedCalc Software, Ostend, Belgium) software.

\section{Subjective image quality analysis}

Five type 2 phantoms were used. Each phantom was positioned in a standardized fashion, with its base parallel to the horizontal plane. As no articulation was possible between the base and the skull, the position of the skull was standardized on all devices. All 5 phantoms were exposed to all 3 devices. Thus, in total, 15 lateral cephalographs (5 phantoms $\times 3$ devices) were obtained.

The images were organized in 5 triads. Each triad consisted of the images of the same phantom obtained with the 3 devices, anonymized and arranged in a random sequence (Fig. 4). Seven oral radiologists evaluated the images. In each triad, 1 image was chosen as "best," one as "intermediate," and the last as of "inferior" quality, with regard 
to the visual appearance as measured by the sharpness of the images and the visibility of bone, teeth, and soft-tissue simulator. No changes in brightness and contrast were

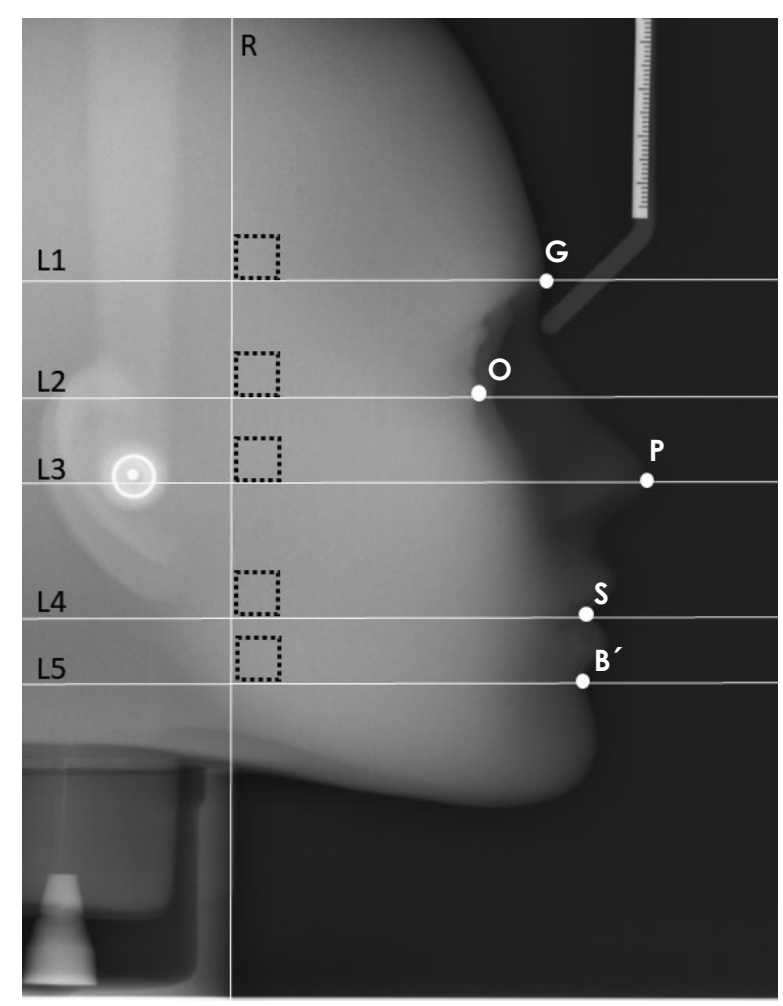

Fig. 3. Five lines (L1-L5) are drawn perpendicular to line $\mathrm{R}$ and parallel to the horizontal plane with reference to the cephalometric landmarks on the lateral cephalographs of the type 1 phantom. G: glabella, O: orbitale, $\mathrm{P}$ : pronasale, S: stomion, B': B point. The reference lines are used to delimit the evaluated regions of interest (dotted squares). observed. The data obtained were tabulated and described qualitatively.

\section{Cephalometric analysis}

The 15 lateral cephalographs obtained for the subjective image quality analysis were anonymized, randomly organized, and subjected to cephalometric analysis. Three oral radiologists with training in orthodontics and experience in cephalometric analyses performed the evaluation together and, via consensus, marked the cephalometric landmarks for the McNamara analysis using Radiocef software (RadioMemory, Belo Horizonte, MG, Brazil). The contrast and brightness could be manipulated while evaluating the radiographs. In the McNamara cephalometric analysis, 9 measurements were considered: the relationship between the maxilla and the cranial base, the relationship between the mandible and the cranial base, the effective midfacial length, the effective mandibular length, the lower anterior facial height, the mandibular plane angle, the facial axis angle, the anteroposterior relationship of the upper incisor with the maxilla, and the anteroposterior relationship of the lower incisor with the mandible (Table 1), excluding measurements associated with the soft tissue and airway. Fifteen days after the initial evaluation, $60 \%$ of the images were reevaluated.

One-way ANOVA was applied with the Tukey post-hoc test to compare the 3 detectors with regard to the values obtained in the cephalometric analyses. $P$-values $<0.05$ were considered to indicate statistical significance. The ICC was used to assess intra-examiner agreement.
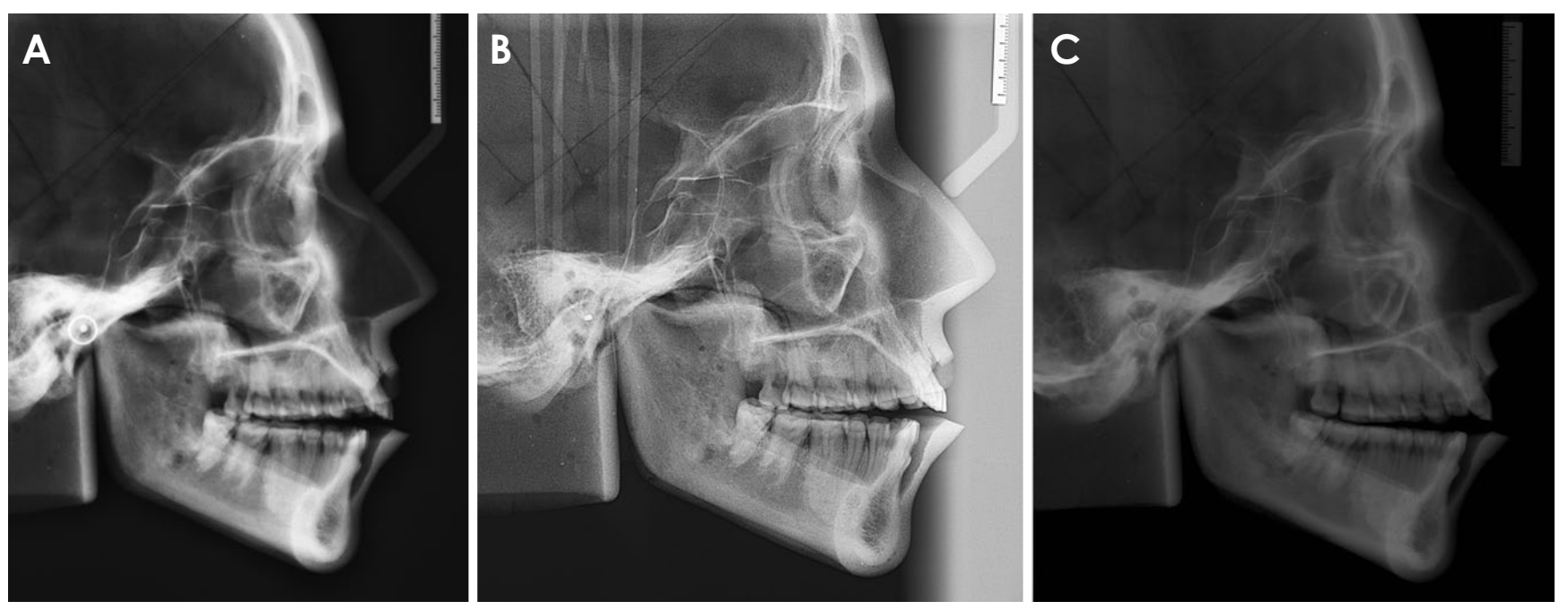

Fig. 4. Lateral cephalographs of the type 2 phantom for subjective analysis. The images are acquired with a charge-coupled device (CCD, A), complementary metal oxide semiconductor (CMOS, B), and cadmium telluride (CdTe, C) detector. 


\section{Dentoskeletal analysis}

Based on the values obtained in the McNamara analysis, for all of the measurements taken, each phantom was classified as having a certain facial configuration (Table 2). The classification was performed for each measurement, which was compared to the range of reference values (the normal range). Measurements with values higher than the reference range were assigned a score of 3 , measurements within the reference range (the range of normality) were assigned a score of 2 , and measurements lower than the reference range were assigned a score of 1 .

The data regarding the classifications of the 5 type 2 phantoms, obtained using the 3 different detectors, were recorded. The analyses of dentoskeletal configuration were described qualitatively.

\section{Dosimetry analysis}

Five triads of thermoluminescent dosimeters made of

Table 1. McNamara analysis measurements used for cephalometric evaluation

\begin{tabular}{ll}
\hline \multicolumn{1}{c}{ Measurement } & \multicolumn{1}{c}{ Interpretation } \\
\hline A-N perpendicular & $\begin{array}{l}\text { Relationship between the maxilla and } \\
\text { the cranial base } \\
\text { Relationship between the mandible and } \\
\text { the cranial base } \\
\text { Effective midfacial length }\end{array}$ \\
Co-A & $\begin{array}{l}\text { Effective mandibular length } \\
\text { Co-Gn }\end{array}$ \\
ANS-Me & $\begin{array}{l}\text { Mandibular anterior facial height } \\
\text { Facial axis angle }\end{array}$ \\
(Po-Or).(Go-Me) & $\begin{array}{l}\text { Anteroposterior relationship of UI with } \\
\text { the maxilla } \\
\text { UI-A vertical }\end{array}$ \\
Anteroposterior relationship of LI with \\
the mandible
\end{tabular}

UI: upper incisor, LI: lower incisor lithium fluoride (Thermo Fisher Scientific Inc., Waltham, MA, USA) were positioned in the type 2 phantom in the orbital regions (on both sides, near and opposite the $\mathrm{x}$-ray source), the parotid gland regions (near and opposite the $\mathrm{x}$-ray source), and the thyroid gland region. The phantom with the dosimeters was positioned in the device in such a way as to simulate patient positioning and was exposed to radiation 10 times consecutively, with a 3-minute interval between exposures. To calculate the background radiation, a triad of dosimeters was positioned outside the acquisition room during the phantom exposure. This procedure was repeated for 3 days.

The dosimeters were read in a Harshaw Thermoluminescent reader (Model 2000; Thermo Fisher Scientific Inc.). Averages of the triad values for each region were obtained, including those from the dosimeters that remained outside the room for background calculation. To measure the dose on the surface of the organ, the following calculation was used:

Dose on the organ surface $=$ mean dose of the dosimeters of the regionmean dose of the background dosimeters

The total surface dose for each detector was obtained by summing the doses of the 5 regions in which the dosimeters were positioned. As 10 exposures were performed, the total surface dose was divided by 10 to obtain the dose for each organ for each exposure.

Statistical analysis was performed to compare the absorbed dose values via 1-way ANOVA with the Tukey posthoc test.

\section{Results}

\section{Objective analysis of image quality}

Table 3 shows the image noise values for each detector. Regarding the mean noise levels, a significant difference

Table 2. Dentoskeletal classification based on the values obtained in the cephalometric analysis

\begin{tabular}{|c|c|c|c|}
\hline Measurement & Higher & Reference & Lower \\
\hline Relationship between the maxilla and the cranial base & Protrusion & Normal & Retrusion \\
\hline Relationship between the mandible and the cranial base & Protrusion & Normal & Retrusion \\
\hline Effective midfacial length & Large & Normal & Small \\
\hline Effective mandibular length & Large & Normal & Small \\
\hline Lower anterior facial height & Increased & Normal & Reduced \\
\hline Mandibular plane angle & Vertical growth & Normal & Horizontal growth \\
\hline Facial axis angle & Horizontal growth & Normal & Vertical growth \\
\hline Anteroposterior relationship of upper incisor with the maxilla & Protruded & Normal & Retruded \\
\hline Anteroposterior relationship of lower incisor with the mandible & Protruded & Normal & Retruded \\
\hline
\end{tabular}


was observed among the 3 detectors $(P<0.05)$. The CdTe detector presented the least noise, followed by the CCD and CMOS detectors. The ICC for the noise measurement was 0.9964 .

\section{Subjective image quality analysis}

The subjective ratings of the overall image quality for the different detectors are presented in Table 4. The images obtained with the CdTe detector were rated as the best in $62.9 \%$ of the evaluations, followed by the CCD and the CMOS images. The CdTe detector was chosen as the best 2 times more than the CCD and 4 times more than the CMOS detector. The CMOS detector most commonly

Table 3. Mean image noise values for each tested detector

\begin{tabular}{lc}
\hline Detector & Noise \\
\hline CCD & $1.84 \pm 0.52^{\mathrm{b}}$ \\
CMOS & $2.59 \pm 0.31^{\mathrm{c}}$ \\
CdTe & $1.48 \pm 0.18^{\mathrm{a}}$ \\
\hline
\end{tabular}

Different letters indicate statistically significant differences (as determined using analysis of variance, with a significance level of 5\%). CCD: chargecoupled device, CMOS: complementary metal oxide semiconductor, CdTe: cadmium telluride

Table 4. Subjective rating of the overall image quality by detector, according to the evaluators' preference

\begin{tabular}{lccc}
\hline Detector & Best & Intermediate & Inferior \\
\hline CCD & $8(22.9 \%)$ & $15(42.8 \%)$ & $12(34.3 \%)$ \\
CMOS & $5(14.2 \%)$ & $10(28.6 \%)$ & $20(57.1 \%)$ \\
CdTe & $22(62.9 \%)$ & $10(28.6 \%)$ & $3(8.6 \%)$ \\
\hline Total & 35 & 35 & 35 \\
\hline
\end{tabular}

CCD: charge-coupled device, CMOS: complementary metal oxide semiconductor, CdTe: cadmium telluride presented the image subjectively chosen as being of inferior quality $(57.1 \%)$. Regarding the CCD detector, the data from the evaluators did not provide a clear rating regarding the quality of its images.

\section{Cephalometric analysis}

Table 5 shows the mean values of each measure evaluated in the cephalometric analysis for the images of the 3 detectors studied. No statistically significant difference $(P>0.05)$ among the different detectors was observed in any of the evaluated measurements. The ICC for intra-examiner agreement was 0.9988 .

\section{Dentoskeletal analysis}

Perfect agreement was observed among the dentoskeletal configurations evaluated based on the images of the different detectors, for all phantoms, in all 3 comparisons (CCD with CMOS, CCD with CdTe, and CMOS with CdTe).

\section{Dosimetry analysis}

The radiation dose for each region and the total dose, for each detector, are presented in Table 6 . No statistically significant difference in radiation dose was observed for the exposure protocols for the CCD and CdTe detectors in the orbit (opposite the source, $P>0.05$; near the source, $P>0.05$ ), parotid gland (opposite the source, $P>0.05$ ), and thyroid $(P>0.05)$. However, the protocol used for the CMOS detector yielded higher doses than the protocols of the $\mathrm{CCD}(P<0.05)$ and $\mathrm{CdTe}(P<0.05)$ detectors for these regions.

The radiation dose in the parotid gland near the source showed a statistically significant difference among all of the detectors $(P<0.05)$, with the lowest dose associated with the CCD receptor.

Although no statistically significant difference in total ra-

Table 5. Mean measurements in the cephalometric analysis by detector

\begin{tabular}{|c|c|c|c|c|}
\hline Measurement & $\mathrm{CCD}$ & CMOS & $\mathrm{CdTe}$ & $P$-value \\
\hline A-N perpendicular (mm) & $7.1 \pm 2.9$ & $7.9 \pm 2.1$ & $8.5 \pm 2.7$ & $P>0.05$ \\
\hline Pg-N perpendicular $(\mathrm{mm})$ & $5.7 \pm 12.4$ & $8.5 \pm 8.0$ & $8.9 \pm 8.0$ & $P>0.05$ \\
\hline $\mathrm{Co}-\mathrm{A}(\mathrm{mm})$ & $92.3 \pm 2.6$ & $93.8 \pm 3.6$ & $93.4 \pm 4.54$ & $P>0.05$ \\
\hline Co-Gn (mm) & $121.3 \pm 4.3$ & $123.6 \pm 7.7$ & $123.3 \pm 7.0$ & $P>0.05$ \\
\hline $\operatorname{ANS}-\mathrm{Me}(\mathrm{mm})$ & $71.0 \pm 5.5$ & $72.5 \pm 5.7$ & $71.6 \pm 5.1$ & $P>0.05$ \\
\hline$(\mathrm{Po}-\mathrm{Or}) \cdot(\mathrm{Go}-\mathrm{Me})\left({ }^{\circ}\right)$ & $24.4 \pm 7.7$ & $22.6 \pm 6.0$ & $23.0 \pm 7.9$ & $P>0.05$ \\
\hline$(\mathrm{Ba}-\mathrm{N}) \cdot(\mathrm{Ptm}-\mathrm{Gn})\left({ }^{\circ}\right)$ & $-1.9 \pm 6.4$ & $-0.2 \pm 5.8$ & $-0.5 \pm 6.2$ & $P>0.05$ \\
\hline U1-A vertical (mm) & $7.2 \pm 2.1$ & $8.4 \pm 2.4$ & $7.6 \pm 1.8$ & $P>0.05$ \\
\hline L1-(A-Pg) (mm) & $5.1 \pm 3.0$ & $5.7 \pm 3.3$ & $5.3 \pm 3.3$ & $P>0.05$ \\
\hline
\end{tabular}

CCD: charge-coupled device, CMOS: complementary metal oxide semiconductor, CdTe: cadmium telluride 
Table 6. Mean radiation dose (mGy) detected in the tested regions by detector

\begin{tabular}{|c|c|c|c|c|}
\hline Region & CCD & CMOS & $\mathrm{CdTe}$ & $P$-value \\
\hline Orbit opposite the X-ray source & $0.0043^{\mathrm{a}}$ & $0.0147^{\mathrm{b}}$ & $0.0017^{\mathrm{a}}$ & $P<0.05$ \\
\hline Orbit near the X-ray source & $0.0432^{\mathrm{a}}$ & $0.0939^{\mathrm{b}}$ & $0.0468^{\mathrm{a}}$ & $P<0.05$ \\
\hline Parotid opposite the $\mathrm{X}$-ray source & $0.0028^{\mathrm{a}}$ & $0.0292^{\mathrm{b}}$ & $0.0012^{\mathrm{a}}$ & $P<0.05$ \\
\hline Parotid near the X-ray source & $0.0367^{\mathrm{a}}$ & $0.2977^{\mathrm{c}}$ & $0.0665^{\mathrm{b}}$ & $P<0.05$ \\
\hline Thyroid & $0.0131^{\mathrm{a}}$ & $0.1314^{\mathrm{b}}$ & $0.0173^{\mathrm{a}}$ & $P<0.05$ \\
\hline Total & $0.1001^{\mathrm{a}}$ & $0.5569^{\mathrm{b}}$ & $0.1335^{\mathrm{a}}$ & $P<0.05$ \\
\hline
\end{tabular}

Different letters indicate statistically significant differences (as determined using analysis of variance, with a significance level of 5\%). CCD: charge-coupled device, CMOS: complementary metal oxide semiconductor, CdTe: cadmium telluride

diation dose was observed for the CCD and CdTe detectors $(P>0.05)$, the dose of the protocol used for the CMOS detector was significantly higher than those $\operatorname{doses}(P<0.05)$.

\section{Discussion}

An important requirement of a digital radiographic image detector is the potential for acquiring images of high enough quality to correctly diagnose a condition, with exposure to a radiation dose in accordance with the "as low as reasonably achievable (ALARA)" principle.

Image noise is a cause of degradation of radiographic image quality. Theoretically, to be considered of good quality, an image should have noise minimized as much as possible. In the present study, we observed the least noise in the images obtained with the CdTe detector, followed by the images obtained with the CCD and CMOS detectors, in that order. This can be explained by the fact that the CdTe detector acquires images through the direct detection of X-ray photons. ${ }^{5,6}$ Relative to indirect detectors, direct detectors inherently exhibit less scattering of the image signal, ${ }^{5}$ which results in less noise. Although the CCD and CMOS detectors are of the same type $\mathrm{e}^{1,3}$ - that is, indirect detectors that make use of scintillators - they differ in noise level. This difference can be explained by how the electric charge is read. In the present study, the images acquired with the CMOS detector exhibited more noise than the images obtained with the CCD detector, which aligns with the studies of Sanderink and Miles ${ }^{1}$, Parks et al. ${ }^{7}$ and Gilmore et al. ${ }^{4}$ This might be due to the individual reading of each pixel in CMOS detectors, which causes each to be converted and calibrated separately and may lead to a mismatch.

The subjective preference with regard to visual quality for the images obtained with the CdTe detector may be associated with the lower noise levels - reflected as better overall brightness and contrast - present due to the inher- ent characteristics of direct detection. ${ }^{5}$ Factors associated with the visual acuity of the evaluators and their preferences for brightness, contrast, and image granulation may also have influenced their subjective ratings.

Even though differences in objective quality and visual aspects were observed, the overall quality of the images did not interfere with the cephalometric analysis. The small differences observed in absolute values were not of a magnitude that would lead to a diagnosis being changed. In addition, these minimal variations may be inherent to the subjective process of cephalometric analysis. Shi et al. ${ }^{8}$ compared a CMOS detector to a CCD detector and found that the CMOS images allowed for higher accuracy in the perception of low-contrast structures. However, in the present study, for the proposed diagnostic task, no statistically significant difference was observed between the detectors. This result was similar to findings of the study by Kitagawa et al., ${ }^{2}$ in which the authors compared images obtained with $\mathrm{CCD}$ and CMOS detectors in the context of different diagnoses in dentistry, such as the detection of proximal carious lesions; the evaluation of the cortical bone, root canal space, root apex, and periodontal ligament space; and the evaluation of the presence of an endodontic instrument.

The exposure parameters indicated by the manufacturers for medium-size adults can explain the difference in radiation doses observed for each detector. The similar tube current values observed for the exposures with the CCD and CdTe detectors resulted in similar doses for those detectors. Consequently, the higher current and peak kilovoltage used for the exposure of the CMOS detector resulted in a higher radiation dose. Thus, the difference in doses obtained for the different detectors was more closely associated with the parameters used than with the characteristics of the detectors themselves. As the CdTe detector presents good linearity between incident photons and signal input, ${ }^{9,10}$ it is associated with a higher sensitivity, which can be observed 
in the higher density of the images obtained with this detector (e.g., Fig. 2). Therefore, future studies must be considered to evaluate the possibility of obtaining images with exposure parameters even lower than those recommended by the manufacturer, with similar image quality. Notably, the purpose of using the parameters recommended by the manufacturer of each device was to obtain images with the quality needed for diagnosis instead of standardizing the exposure parameters, which could have yielded images of inferior quality. This standardization would likely still allow the approximation of the dose values, but it would not correctly reflect the clinical situation and could interfere negatively with the image quality. Therefore, images could be rated as "inferior" due to the incorrect exposure parameters instead of the quality of the detector, the latter of which was the focus of the present study. In this way, such standardization could bias the results; thus, it was decided to not standardize the exposure parameters, but rather to use the most appropriate parameters for each detector.

It is worth mentioning that, although the average parameters were selected in this in vitro study, in clinical practice the exposure parameters should be selected according to the characteristics of the patient.

In conclusion, although the differences in detectors were not reflected in the diagnosis (as the CCD, CMOS, and CdTe detectors performed similarly in the diagnostic task evaluated), the CdTe detector yielded images of superior objective and subjective quality compared to those obtained using the other detectors. Thus, considering these findings, it is recommended to use the CdTe detector in clinical practice.

\section{Conflicts of Interest: None}

\section{Acknowledgements}

This material was part of the PhD thesis of PM Queiroz.

\section{References}

1. Sanderink GC, Miles DA. Intraoral detectors. CCD, CMOS, TFT, and other devices. Dent Clin North Am 2000; 44: 24955 .

2. Kitagawa H, Scheetz JP, Farman AG. Comparison of complementary metal oxide semiconductor and charge-coupled device intraoral X-ray detectors using subjective image quality. Dentomaxillofac Radiol 2003; 32: 408-11.

3. van der Stelt PF. Principles of digital imaging. Dent Clin North Am 2000; 44: 237-48.

4. Gilmore J, Weldon J, Lares M. CMOS technology for digital dental imaging. BioPhotonics [Internet]. 2010 Apr [cited 2020 Mar 13]. Available from: https://www.photonics.com/Articles/ CMOS_technology_for_digital_dental_imaging/a42008.

5. Shvydka D, Jin X, Parsai EI. Performance of large area thinfilm CdTe detector in diagnostic X-Ray imaging. Int J Med Phys Clin Eng Radiat Oncol 2013; 2: 98-109.

6. Takahashi T, Watanabe S. Recent progress in CdTe and CdZnTe detectors. IEEE Trans Nucl Sci 2001; 48: 950-9.

7. Parks ET, Williamson GF. Digital radiography: an overview. J Contemp Dent Pract 2002; 3: 23-39.

8. Shi XQ, Benchimol D, Näsström K. Comparison of psychophysical properties of two intraoral digital sensors on low-contrast perceptibility. Dentomaxillofac Radiol 2013; 42: 20130249.

9. Ogawa K, Langlais RP, McDavid WD, Noujeim M, Seki K, Okano T, et al. Development of a new dental panoramic system based on a tomosynthesis method. Dentomaxillofac Radiol 2010; 39: 47-53.

10. Katsumata A, Ogawa K, Inukai K, Matsuoka M, Nagano T, Nagaoka $\mathrm{H}$, et al. Initial evaluation of linear and spatially oriented planar images from a new dental panoramic system based on tomosynthesis. Oral Surg Oral Med Oral Pathol Oral Radiol Endod 2011; 112: 375-82. 